\title{
Recognition of Isolated Handwritten Oriya Numerals using Hopfield Neural Network
}

\author{
Pradeepta K Sarangi \\ School of Computer Science \\ Apeejay Institute of \\ Technology \\ Greater Noida,U.P, India
}

\author{
Ashok K Sahoo \\ Department of Computer \\ Science and Engineering \\ Sharda University, \\ Greater Noida, U.P, India
}

\author{
P Ahmed \\ Department of Computer \\ Science and Engineering \\ Sharda University, \\ Greater Noida, U.P, India
}

\begin{abstract}
Designing an automatic pattern recognition system is a challenging task. However, despite the design challenges, its enormous application potentials have attracted the attention of researchers and developers over the last four to five decades. Design of recognition systems for handwritten character applications has been a subject of intensive research, and the search is still on for a robust technique capable of dealing with natural variations in handwritten characters. In this paper the performance of Hopfield neural network (HNN) model in recognizing the handwritten Oriya (an Indian language) digits is addressed. The implementation has been carried out in two different ways. In first case, 290 test patterns (29 elements of each classes 0-9) created by different persons in Microsoft Paint were presented to the network in image form of $12 \times 12$. It is found that the network recognized $97.95 \%$ of the input characters correctly even if $40 \%$ of the input characters were having a significant level of noise. In the second experiment, the inputs were the collected handwritten characters in image format.A total of 1500 different input patterns were fed to the network sequentially and $95.4 \%$ recognition accuracy is achived. All the activities such as preprocessing of data (image cropping, resizing digitization and implementations) have been carried out using MATLAB.
\end{abstract}

\section{General Terms}

Pattern Recognition, Hopfield Neural Network

\section{Keywords}

Oriya Numerals, Handwritten Character Recognition.

\section{INTRODUCTION}

Computer is a wonderful invention for the society. Since its invention, it has not only touched every aspect of human activities, but also has tremendously enhanced its own capability to fulfill the human requirements with greater ease and better accuracy. Automatic analysis of handwriting or hand written symbols/characters is one of the human requirements that have been a subject of intensive research for the last few decades and it is still far from the perfection. Such requirements gave birth to novel area of knowledge which is termed as machine learning. Machine learning describes the study of machines that can adapt to their environment and learn from observations. One such example is the conversion of handwritten documents into computer manipulable form and it is such an area which is still unexplored and need researcher's attention. Analysis that involves computer recognition of handwritten characters uses pattern recognition methods. In pattern recognition, the machine learns from a set of patterns and once the learning process is over, then it identifies an unknown pattern based on the learnt patterns.

Oriya is a regional language derived from the Devanagari script commonly used in north-eastern states of India. It is mainly spoken in the states of Orissa and West Bengal in India. Oriya is one of the many official languages of India. It is the official language of Orissa accounting for over 40 million people and the second official language of Jharkhand (another state in India).

In Oriya language ten numerals are shown in figure-1 below.

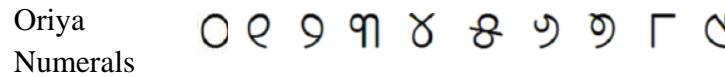 \\ Equivalent \\ English \\ $\begin{array}{llllllllll}0 & 1 & 2 & 3 & 4 & 5 & 6 & 7 & 8 & 9\end{array}$ \\ Numerals}

Figure 1. Oriya numerals (0-9) and equivalent English numerals.

\section{RELATED WORKS}

Many researchers have worked on recognition of Oriya characters. Very few works were found on handwritten Oriya numerals [1] [2] [3]. In this section, we present some works related to handwritten Oriya numerals recognition using different techniques and datasets carried out by different researchers followed by a summary on works done on other Oriya characters.

\subsection{Recent works on handwritten Oriya numerals}

Recognition of handwritten Oriya numerals along with five other popular Indian scripts is reported in [1] . The authors have proposed a modified quadratic classifier based scheme towards the recognition of off-line handwritten numerals of six popular Indian scripts such as Devnagari, Bangla, Telugu, Oriya, Kannada and Tamil. They have used 22546, 14650, 2220, 5638, 4820, and 2690 numeral samples of Devnagari, Bangla, Telugu, Oriya, Kannada 
and Tamil scripts, respectively. Here, the authors have used two sets of features, 64 dimensional features for highspeed recognition and 400 dimensional features for highaccuracy recognition. A five-fold cross validation technique has been used for result computation and accuracy of $99.56 \%, 98.99 \%, 99.37 \%, 98.40 \%, 98.71 \%$ and $98.51 \%$ is obtained from Devnagari, Bangla, Telugu, Oriya, Kannada, and Tamil scripts, respectively. The authors have computed accuracy of the individual numerals also of the six scripts. The lowest accuracy that was observed in Devnagari, Bangla, Telugu, Oriya, Kannada and Tamil scripts for numeral two $(99.11 \%)$, one (97.74\%), six (97.35\%), seven $(96.19 \%)$, seven $(97.46 \%)$ and six $(96.53 \%)$, respectively, when 400 dimensional feature vectors were considered. The authors report that in case of Oriya script the main confusing numerals were numerals (seven) and (two). Their experiment shows that these two numerals caused confusion in $0.42 \%$ cases.

An HMM based recognition scheme for handwritten Oriya Numerals is reported in [2]. The authors have used the database of isolated handwritten Oriya numerals [3] consisting of 5970 samples collected from real-life documents like postal mail pieces, job application forms etc. The above database is exclusively divided into training and test sets. The training and test databases consist of respectively 4970 and 1000 images of handwritten numerals of all the 10 classes. The authors have used the features indicating the shape, size and position of a digital curve with respect to the numeral image. From these numeral images, 15231 horizontal and 11014 vertical strokes have been extracted from the training set whereas 3078 horizontal and 2204 vertical stokes have been extracted from the test set. The authors report the achievement of $90.50 \%$ correct recognition rate on the test set and $95.89 \%$ on the training set.

Recognition of off-line unconstrained Oriya handwritten numerals is reported in [4]. The authors have applied their scheme on 3850 numerals obtained from 385 different individuals of different professions like school, college and university students and teacher, government employees, businessmen etc. The authors have used two sets of features for Oriya numeral recognition. For high-speed recognition the authors have used 64 dimensional features, and for high accuracy recognition they have used 400 dimensional features. The overall recognition accuracy of their scheme is about $90.38 \%$ with a rejection rate of $1.84 \%$. The authors have also used 400 dimensional features set in quadratic classifier and obtained a success rate of $94.81 \%$ with a rejection rate of $1.3 \%$ from quadratic classifier. The authors also have reported that numeral 'four' has the highest recognition rate $(99.01 \%)$ and maximum confusing numeral pair is 'six' and 'seven' followed by seven and two. They have also analyzed error versus rejection rate in each of the classifiers and reported that from NN classifier they observed $2.66 \%$ error while the rejection rate was $15.38 \%$. From the quadratic classifier with 64 (400) dimensional features, they observed $1.98 \%(0.97 \%)$ errors while the rejection rate was $10.48 \%(14.20 \%)$, respectively.

\subsection{Works on other Oriya characters}

Multifont Oriya character recognition using curvelet transform is reported in [5]. Ten popular Oriya fonts have been used for the purpose of character recognition. The authors have used curvelet transform for recognition which is done using curvelet coefficients. The proposed method is simple and extracts effectively the features in target region, which characterize better and represent more robustly the characters. The experimental results validate that the proposed method improves greatly the recognition accuracy and efficiency over other traditional methods.

Performance comparison of SVM and $K$-NN for Oriya character recognition is reported in [6]. The authors mainly discuss feature vector-based classification methods, which have prevailed upon structural methods, especially in printed character recognition. These methods include $K$ Nearest Neighborhood and Support Vector Machines. The authors have used printed Oriya characters as data set and report $98.9 \%$ accuracy for SVM and $96.47 \%$ accuracy for KNN over a test data set of 10000 .

Oriya character recognition using neural networks is reported in [7]. The authors have used Back propagation neural network for printed Oriya characters. The authors discuss briefly and show how they have helped build new OCRs for the purpose of recognizing Oriya script. The authors have used the Back propagation Neural Network for efficient recognition where the errors were corrected through back propagation and rectified neuron values were transmitted by feed-forward method in the neural network of multiple layers, i.e. the input layer, the output layer and the middle layer or hidden layers.

An approach for Bilingual (English - Oriya) script identification and recognition in a printed document is reported in [8]. In this work the authors have tried to distinguish between the English and Oriya documents through horizontal projection profiles for intensity of pixels in different zone along with the line height and the number of characters present in that line. The authors have tried to recognize the Oriya script and Roman script with two separate training sets using Support Vector Machines. The authors have performed experiments with different types of images such as normal, bold, thin, small, big, etc., having varied sizes of Oriya and Roman characters. The training and testing set consists of more than 10, 000 samples. The authors have used horizontal histogram for line distinction belonging to different scripts. Accuracy of $99.8 \%$ (for bold and big fonts) is reported.

An Efficient Bilingual Optical Character Recognition (English-Oriya) System for Printed Documents is reported in [9].This paper describes the character recognition process for printed documents containing English and Oriya texts. The authors have tried to distinguish between the English and Oriya documents through histogram (both 
horizontal and vertical) and aspect ratio. They have tried to recognize the Oriya scripts using structural analysis.

All these observations reveal that impressive research works are going on Oriya characters but, still it is far from its perfection. To the best of our information, no effective research work is done on recognition of handwritten Oriya numerals, and almost no work has been carried out for handwritten Oriya numerals using Hopfield neural network. This research work focuses on the assessment of recognition performance of Hopfield neural network in handwritten Oriya numerals.

\subsection{The Data Base}

The survey on computer recognition of Oriya characters shows that the researchers have used different data sets in their research. This indicates that in case of Oriya character recognition no standard data set exists. However, the authors in [1] have reported the ongoing development of a datasets for six different Indian scripts including Oriya. Authors in [2] have reported the existence of a database of isolated handwritten Oriya numerals consisting of 5970 samples collected from real-life documents like postal mail pieces, job application forms etc.

\section{THE HOPFIELD NETWORK}

Singh et.al [10] describes Hopfield network as Hopfield associative memory, which is a special kind of recurrent neural network. It can be used as associative memory i.e. a memory that is addressed through its contents.

If a pattern (even if distorted) is presented to an associative memory as input, it returns a stored pattern which matches the best with the input pattern. The matching may not be always perfect. An associative memory may also return a stored pattern that is not similar to the presented one and the effect is that noisy input can also be recognized.

In the Hopfield model, each neuron has two states. The ON state of the neuron is denoted by the output +1 , and the OFF state is represented by -1 . There are two phases of the operation of the Hopfield network:

\section{Storage phase and Retrieval phase.}

In the storage phase, $\mathrm{N}$-dimensional characters are stored in the memory. These characters can be retrieved during retrieval phase when these characters are presented to the network as test vectors [10].

The Hopfield associative memory stores ' $M$ ' bipolar patterns $A_{1}, A_{2} \ldots A_{M}$ by summing them together ' $M$ ' outer products. The mathematical equations for storing and retrieval of patterns in a Hopfield associative memory described by [11] are given in equation 1 to 5

$$
T=\sum_{i=1}^{M}\left[A_{i}^{T}\right]\left[A_{i}\right]
$$

where, $T=\left[t_{i j}\right]$ is a $(p \times p)$ is connection matrix and

$$
A_{i} \in\{-1,1\}^{p}
$$

The recall equation is given by

$$
a_{j}^{\text {new }}=f\left(a_{i} t_{i j}, a_{j}^{\text {old }}\right) \forall j=1,2, \ldots, p
$$

where $A_{i}=\left(a_{1}, a_{2}, \ldots, a_{p}\right)$ and the two parameter bipolar threshold function is

$$
f(\alpha, \beta)=\left\{\begin{array}{c}
+1, \text { if } \alpha>0 \\
\beta, \text { if } \alpha=0 \\
1, \text { if } \alpha<0
\end{array}\right.
$$

The proximity of the noisy pattern to the stored patterns is measured by calculating the Hamming distance. The Hamming distance (HD) of a vector $\mathrm{X}$ from $\mathrm{Y}$, given $\mathrm{X}=\left(\mathrm{x}_{1}, \mathrm{x}_{2} \ldots, \mathrm{x}_{\mathrm{n}}\right)$ and $\mathrm{Y}=\left(\mathrm{y}_{1}, \mathrm{y}_{2} \ldots, \mathrm{y}_{\mathrm{n}}\right)$ is given by

$$
H D(x, y)=\sum_{i=1}^{n}\left|x_{i}-y_{i}\right|
$$

\section{DATA PREPROCESSING AND IMPLEMENTATION DESIGN.}

This research deals with isolated handwritten Oriya numerals. As mentioned before, no standard data set is available for handwritten Oriya numerals. Hence we have created our own data set for this research. Since handwritten characters of different persons are different, we collected handwritten characters from different persons on a plain paper. The respondents were of different qualifications, age groups and professions. Each respondent was asked to write each numeral five times as shown in the figure-2. A total of 1500 different numerals (150 numerals of each class) were collected from 30 persons. 


\begin{tabular}{|c|c|c|c|c|c|c|c|c|c|c|}
\hline \multirow{2}{*}{\multicolumn{11}{|c|}{ Person:1 }} \\
\hline & & & & & & & & & & \\
\hline \multicolumn{6}{|c|}{ Name: S.P. Penda } & \multicolumn{2}{|c|}{ Age: 38} & & & \\
\hline \multicolumn{6}{|c|}{ Qualification:Grondiatton } & \multicolumn{3}{|c|}{ Occupation: $50 b$} & 8 & 9 \\
\hline 1 & 0 & $e$ & 9 & m & $\gamma$ & 2 & 2 & 3 & $\Gamma$ & $N$ \\
\hline 2 & 0 & e & 9 & m & $\gamma$ & $x$ & כ & 3 & $r$ & $N$ \\
\hline 3 & 0 & e & 9 & n & $\gamma$ & $x$ & 2 & 3 & $\Gamma$ & $N$ \\
\hline 4 & 0 & $e$ & 9 & $\infty$ & $\gamma$ & $x$ & 2 & 3 & $\Gamma$ & 4 \\
\hline 5 & 0 & e & 9 & on & $\gamma$ & $x$ & 2 & 3 & $\Gamma$ & $N$ \\
\hline \multicolumn{11}{|c|}{ Person:2 } \\
\hline \multicolumn{11}{|c|}{$\begin{array}{l}\text { Name:DR-PRASANT SARANGI Age: } 34 \\
\text { Qualification:Ph.D } \\
\text { Occupation: }\end{array}$} \\
\hline & 0 & 1 & 2 & 3 & 4 & 5 & 6 & 7 & 8 & 9 \\
\hline 1 & 0 & e & 9 & $n$ & $\gamma$ & 8 & $\supset$ & 69 & $r$ & $W$ \\
\hline 2 & 0 & e & 9 & $m$ & $\gamma$ & $R$ & $>$ & 9 & $r$ & $W$ \\
\hline 3 & 0 & e & 9 & $n$ & $\gamma$ & 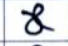 & $>$ & 9 & $r$ & $W$ \\
\hline 4 & 0 & e & 9 & $n$ & $\gamma$ & $a$ & 3 & (9) & $r$ & $W$ \\
\hline 5 & 0 & 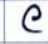 & 9 & $n$ & $\gamma$ & $\alpha$ & 7 & 9 & $r$ & $W$ \\
\hline
\end{tabular}

Figure 2. Sample data set collected from two persons

The input patterns used in this research are images in (.bmp) format. The data collected were scanned and isolated characters were extracted (figure-3).

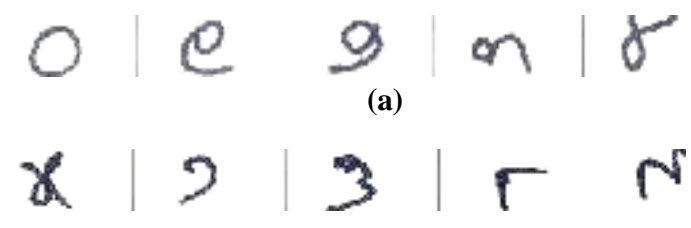

(b)

Figure 3. Sample isolated characters extracted from data set. (a) Oriya numerals 0-4 (b) Oriya numerals 59

The scanned isolated characters are of different dimensions and are not suitable at this stage to be used as it is. Hence, all the characters have under gone different morphological operations using MATLAB functions. The pre-processing steps used in this research are as below:

Step 1: Isolated character extraction

Step 2: Conversion into the negative of the character

Step 3: Thinning the image

Step 4: Image cropping and resizing

(All the rows and columns having only 'zeros' as elements were removed and then the images were resized to $12 \mathrm{X} 12$ size.)

Once the pre-processing was over, the entire data set was divided into two categories. First category was target patterns consisting of 20 data elements of all numerals. The rest were used as test patterns. A sample of the target patterns is given in figure-4(a) and 4(b).

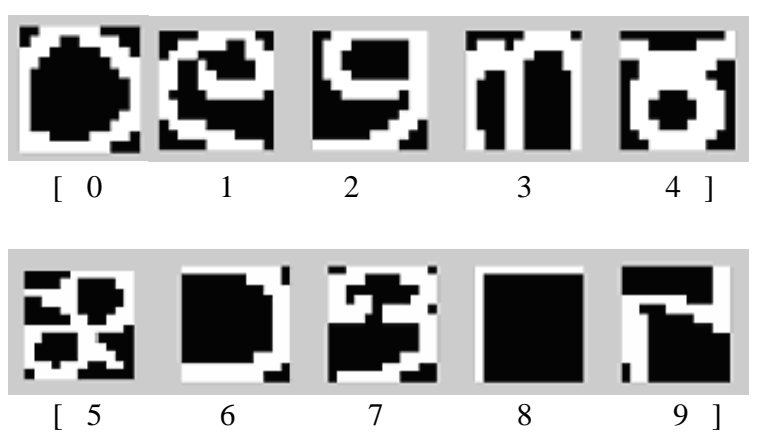

Figure 4. Target patterns: Oriya numerals 0-9

All the experiments have been carried out using MATLAB-7 on a standard Pentium-IV computer with Windows XP as operating system.

\section{IMPLEMENTATION DETAILS AND RESULTS ANALYSIS}

In the case of a Hopfield model, once the network is created by supplying target vectors, stable equilibrium points at these target vectors are stored in the network. Hence, there is no need to perform iterative training on it. That is because Hopfield network learn patterns in a oneshot style.

In this research, we have created the network by providing the target vectors as shown in figure-4. Once the network is created, the recognition process has been carried out in two different types of implementations.

\subsection{Implementation-I}

For this implementation, the data set has been created in Microsoft Paint by various persons. Each character was pre-processed and then presented to the network in image form. The program converts all binary matrices to bipolar form. A total of 290 characters were used to test the recognition ability of HNN.

\subsection{Observations}

It was not surprising when the system recognized correctly most of the test patterns very similar to the target patterns. However, it was surprising that the system recognized the input characters having a significant level of noise (created manually). Figure-5 shows three distorted samples of input pattern 'one' and corresponding network output. The input character is having different levels of noise. In figure-5(i), the noise level is $10.4 \%$ ( 15 bits are swapped), in figure5(ii), the noise level is $13.8 \%$ (20 bits are swapped), in figure 5(iii), the noise level is $17.3 \%$ (25 bits are swapped). In all three cases the network has produced the correct output. 


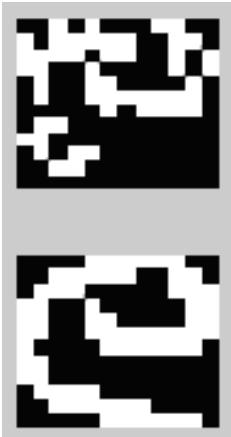

(i)

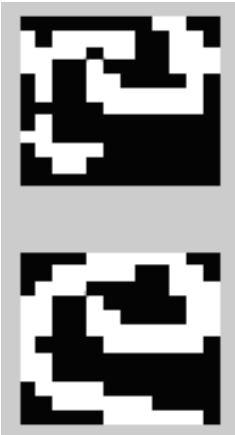

(ii)

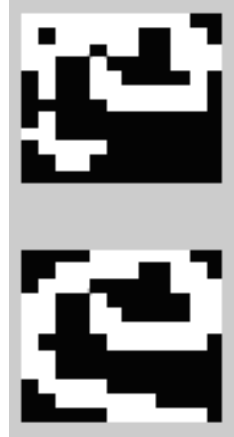

(iii)
Figure 5. Distorted input patterns (Oriya ' 1 ') and corresponding network output.

Similarly, figure-6 and figure-7 represent input patterns for other numerals and their corresponding network output.

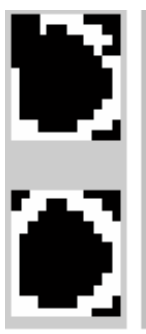

[ 0

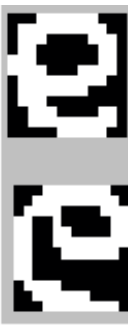

1

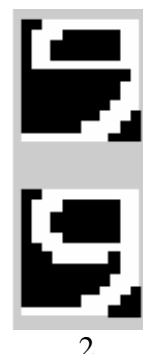

2

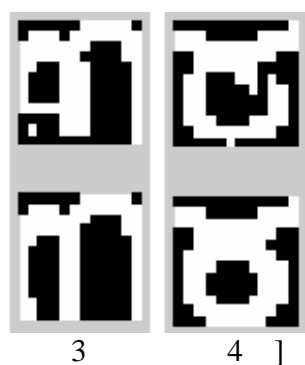

$4]$
Figure 6. Sample input patterns (0-4) and corresponding network output.

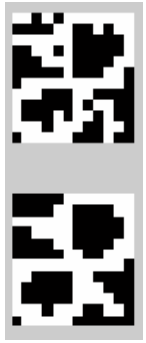

[ 5
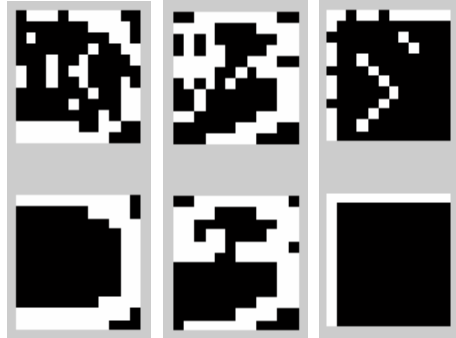

8

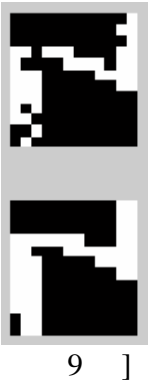

9
Figure 7. Sample input patterns (5-9) and corresponding network output.

In this implementation we got $97.93 \%$ recognition accuracy. Findings of this implementation are summarized in table-1 below.

Table-1: Confusion Matrix

\begin{tabular}{|c|c|c|c|c|c|c|c|c|c|c|c|c|}
\hline $\begin{array}{l}\mathrm{I} \\
\mathrm{n}\end{array}$ & & 0 & 1 & 2 & 3 & 4 & 5 & 6 & 7 & 8 & 9 & $\begin{array}{l}\mathrm{N} . \\
\mathrm{R}\end{array}$ \\
\hline $\mathrm{p}$ & 0 & 29 & 0 & 0 & 0 & 0 & 0 & 0 & 0 & 0 & 0 & 0 \\
\hline $\mathrm{u}$ & 1 & 0 & 29 & 0 & 0 & 0 & 0 & 0 & 0 & 0 & 0 & 0 \\
\hline $\mathrm{t}$ & 2 & 0 & 0 & 27 & 0 & 0 & 0 & 2 & 0 & 0 & 0 & 0 \\
\hline C & 3 & 0 & 0 & 02 & 29 & 0 & 0 & 0 & 0 & 0 & 0 & 0 \\
\hline 1 & 4 & 0 & 0 & 0 & 0 & 29 & 0 & 0 & 0 & 0 & 0 & 0 \\
\hline & 5 & 0 & 0 & 0 & 0 & & 26 & 0 & 0 & 0 & 0 & 0 \\
\hline
\end{tabular}

\begin{tabular}{|c|c|c|c|c|c|c|c|c|c|c|c|c|}
\hline $\mathrm{r}$ & 6 & 0 & 0 & 0 & 0 & 0 & & 29 & 0 & 0 & 0 & 0 \\
\hline$a$ & 7 & 0 & 0 & 0 & 0 & 0 & 0 & & 28 & 0 & 0 & 0 \\
\hline $\mathrm{c}$ & 8 & 0 & 0 & 0 & 0 & 0 & 0 & 0 & & 29 & 0 & 0 \\
\hline $\mathrm{t}$ & 9 & 0 & 0 & 0 & 0 & 0 & 0 & 0 & 0 & & 29 & 0 \\
\hline
\end{tabular}

\subsection{Implementation-II}

In the second experiment, the inputs were the collected handwritten characters (figure-2) in image format (as shown in figure-8).

\section{oleg an $|\gamma| g|3| N$ \\ [ $\left.\begin{array}{llllllllll}0 & 1 & 2 & 3 & 4 & 5 & 6 & 7 & 8 & 9\end{array}\right]$ \\ Figure 8. Sample input characters}

\subsection{Observations}

Here we observed that, the network recognised most of the input patterns even if the pattern holds a significant difference to the corresponding target pattern. However, in some cases, the network produced some eroneous output even if the input pattern is not a completely distorted pattern. Some examples are given in figure- 9 below.

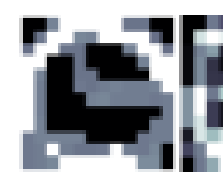

(i)

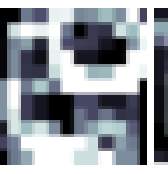

(ii)

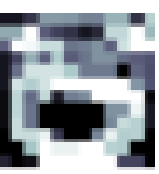

(iii)

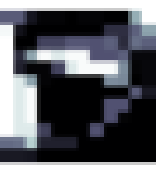

(iv)
Figure 9. Errneous output produced by the network corresponding to 'zero' 'one' 'four' and 'six' respectively

The cause of this errneous out put is that, when the network searches a target pattern based on the Hamming distance, it found two similar values. Hence, the network was confused to exactly identify the target pattern. This problem could be resolved by adding this input pattern to the set of target patterns but, if number of such cases is very large then the netork may exhaust the capacity.

\section{- Confusing Pair of Numerals}

There were some cases where the network was unable to recognize the input character into its proper class. In these cases the network mis-classified the character. For example, the input character 'oriya 2' was recognised as 'Oriya 6', 'Oriya 5' was recognised as 'Oriya 4' and 'Oriya 7' was recognised as 'Oriya 2' as shown in table-2 below. 
Table 2. Confusing Pairs of Numerals

\begin{tabular}{|l|l|l|l|}
\hline Input Character & $\mathbf{\gamma}$ & $\mathbf{X}$ & $\mathbf{0}$ \\
\hline Recognized class & $\mathbf{6}$ & $\mathbf{4}$ & $\mathbf{2}$ \\
\hline Actual Class & $\mathbf{2}$ & $\mathbf{5}$ & $\mathbf{7}$ \\
\hline
\end{tabular}

\section{- Recognition Accuracy}

The recognition accuracy has been calculated in the following way:

(i) All the test patterns are fed to the network for recognition.

(ii) Based on the recognition result, characters producing errneous output are rejected.

The number of recognitions and rejections for each character by our HNN experiment is given in table- 3 .

Table 3: Recognition accuracy of HNN for various Oriya numerals

\begin{tabular}{|l|l|l|l|l|l|}
\hline $\begin{array}{l}\text { Input } \\
\text { numeral }\end{array}$ & $\begin{array}{l}\text { Nu } \\
\text { mbe } \\
\mathrm{r} \text { of } \\
\text { inpu } \\
\mathrm{t}\end{array}$ & $\begin{array}{l}\text { Errneo } \\
\text { us } \\
\text { output }\end{array}$ & $\begin{array}{l}\text { miscl } \\
\text { assifi } \\
\text { cation }\end{array}$ & $\begin{array}{l}\text { Corr } \\
\text { ect } \\
\text { classi } \\
\text { ficati } \\
\text { on }\end{array}$ & $\begin{array}{l}\text { Recogn } \\
\text { ition } \\
\text { accurac } \\
\text { y } \%)\end{array}$ \\
\hline Oriya 0 & 148 & 4 & 2 & 142 & 95.94 \\
\hline Oriya 1 & 148 & 4 & 2 & 142 & 95.94 \\
\hline Oriya 2 & 148 & 3 & 4 & 141 & 95.27 \\
\hline Oriya 3 & 148 & 5 & 0 & 143 & 96.62 \\
\hline Oriya 4 & 148 & 2 & 3 & 143 & 96.62 \\
\hline Oriya 5 & 148 & 5 & 4 & 139 & 93.91 \\
\hline Oriya 6 & 148 & 7 & 2 & 139 & 93.91 \\
\hline Oriya 7 & 148 & 5 & 5 & 138 & 93.24 \\
\hline Oriya 8 & 148 & 5 & 2 & 141 & 95.27 \\
\hline Oriya 9 & 148 & 2 & 2 & 144 & 97.29 \\
\hline \multicolumn{7}{|l}{ Overall recognition accuracy :95.4\% } \\
\hline
\end{tabular}

The recognition accuracy for each numeral is calculated by using the formula:

No. of correct recognitions /No. of characters in test patterns

The confusion matrix for this implementation is given in table- 4 .

\section{Table 4: Confusion Matrix}

\begin{tabular}{|l|c|c|c|c|c|c|c|c|c|c|}
\hline $\begin{array}{l}\text { Numeral } \\
\text { s }\end{array}$ & 0 & 1 & 2 & 3 & 4 & 5 & 6 & 7 & 8 & 9 \\
\hline 0 & 142 & $\mathbf{2}$ & 0 & 0 & 0 & 0 & 0 & 0 & 0 & 0 \\
\hline 1 & $\mathbf{2}$ & 142 & 0 & 0 & 0 & 0 & 0 & 0 & 0 & 0 \\
\hline 2 & 0 & 0 & 141 & 0 & 0 & 0 & $\mathbf{4}$ & 0 & 0 & 0 \\
\hline 3 & 0 & 0 & 0 & 143 & 0 & 0 & 0 & 0 & 0 & 0 \\
\hline
\end{tabular}

\begin{tabular}{|l|l|l|l|l|l|l|r|r|r|c|}
\hline 4 & $\mathbf{3}$ & 0 & 0 & 0 & 143 & 0 & 0 & 0 & 0 & 0 \\
\hline 5 & 0 & 0 & 0 & 0 & $\mathbf{4}$ & 139 & 0 & 0 & 0 & 0 \\
\hline 6 & 0 & 0 & $\mathbf{2}$ & 0 & 0 & 0 & 13 & 0 & 0 & 0 \\
\hline 7 & 0 & 0 & $\mathbf{4}$ & 0 & 0 & 0 & $\mathbf{1}$ & 13 & 0 & 0 \\
\hline 8 & 0 & 0 & 0 & 0 & 0 & 0 & 0 & 0 & 14 & $\mathbf{2}$ \\
\hline 9 & 0 & 0 & 0 & 0 & 0 & 0 & 0 & 0 & $\mathbf{2}$ & 144 \\
\hline
\end{tabular}

It is observed that, the maximum confusing pairs of numerals were "two-six", "five-four" and "seven-two".

\section{CONCLUSION}

Handwritten Oriya character recognition has been implemented using Hopfield model. Recognition accuracy of $95.4 \%$ has been achived. From this research, the following is inferred:

i. Research on recognition of handwritten Oriya numerals is not much explored.

ii. The techniques so far used for handwritten Oriya numeral recognition is limited.

iii. Application of many popular techniques such as Hopfield network is not yet implemented.

iv. No standard data set is available for handwritten Oriya numeral recognition.

v. Hopfield model could be a good recognizer system for handwritten Oriya characters.

vi. Hopfield network produces some erroneous output even if the input pattern is not too much noisy.

vii. The accuracy we obtained is acceptable however it needs to be tested on a large scale database.

\section{ACKNOWLEDGEMENT}

We are thankful to the referees for their valuable comments. We are also thankful to all those persons who have provided us their handwritings for this research.

\section{REFERENCES}

[1] Pal, U., Wakabayashi, T., Sharma, N. and Kimura, F. 2007 Handwritten Numeral Recognition of Six Popular Indian Scripts. Ninth International Conference on Document Analysis and Recognition (ICDAR 2007) Vol 2, ISBN: 0-7695-2822-8

[2] Bhowmik, T. K., Parui, S. K., Bhattacharya, U., Shaw, B. 2006 An HMM Based Recognition Scheme for Handwritten Oriya Numerals. 9th International Conference on Information Technology (ICIT'06) 07695-2635-7/06

[3] Bhattacharya, U. and Chaudhuri, B. B. 2005 Databases for research on recognition of handwritten characters of Indian scripts. Proc. of the 8th Int. Conf. on Document Analysis and Recognition, Seoul, II: 789-793. 
[4] Roy, K., Pal, T., Pal, U. and Kimura, F. 2005 Oriya handwritten Numeral Recognition System, In Proceedings of VIII International Conference on Document Analysis and Recognition, pp. 770-774.

[5] Nigam, S., Khare, A. 2011 Multifont Oriya character recognition using curvelet transform, Communication in Computer and Information Science, Volume 139, part-1, 150-156.

[6] Mohanty, S., Dasbebartta, H. N. 2011 Performance Comparison of SVM and K-NN for Oriya Character Recognition, International Journal of Advanced Computer Science and Applications, Special Issue on Image Processing and Analysis, pp-112-116.

[7] Mishra, S., Nanda, D., Mohanty, S. 2010 Oriya Character Recognition using Neural Networks. Special issue of IJCCT, Vol.2, Issue 2

[8] Mohanty, S., Dasbebartta, H. N. 2010 A Novel Approach for Bilingual (English - Oriya) Script Identification and Recognition in a Printed Document. International Journal of Image Processing (IJIP), Volume (4): Issue (2).
[9] Mohanty, S., Dasbebartta, H. N., Behera, T. K. 2009 An Efficient Bilingual Optical Character Recognition (English-Oriya) System for Printed Documents, VII International Conference on Advances in Pattern Recognition.978-0-76953520/09,IEEE,DOI 10.1109/ICAPR.2009.49

[10] Singh, Y. P., Khare, A., Gupta, A. 2010 Analysis of Hopfield Auto associative Memory in the Character Recognition, International Journal on Computer Science and Engineering, Vol. 02, No. 03, pp 500503.

[11] Rajasekaran,S. Pai, G.A.V. 2007 Neural Networks, Fuzzy Logic and Genetic Algorithms: Synthesis and Applications, PHI, ISBN-978-81-203-2186-1 